\title{
Kafkas Lichtroman „Der Verschollene“", oder: Eine amerikanische Richterwahl im falschen Licht, Amazonenkampf versus Jiu-Jitsu
}

Nun zu den Widersprüchen in der Schluss-Utopie des Kafka'schen Verschollenen, die abschliessend und zusammenfassend noch einmal ins Zentrum der Betrachtung rücken sollen. In ihnen sollte das Erlernen des Goethe'schen Blicks, also die assimilatorische Betonung der eidos-Dominanz, der Welt ihren „paradiesischen“, sozusagen prä-physikalischen Zauber noch einmal zurückgeben. Fanny und Karl finden endlich zueinander, dafür sorgt bei Kafka die Maria-Theresia-âhnliche Hotelköchin, ihrerseits die Geliebte des ungarischen Portiers im „Hotel Occidental“. Doch vor beider anschließenden Himmelfahrt steht, ein harter Übergang, jene Begebenheit, in der, voll naturalistischem Gesellschaftsrealismus, das neueste Amerika noch einmal in ganz erbarmungsloses Licht gestellt wird, und das im Wortsinn. Hier kritisiert der Roman, im Medium der Lichtmetapher und als ein in der Entwicklung der Elektrizitätslehre beschlagener, die allzu versöhnliche, noch naive Licht-Emphase Goethes; er bezieht sozusagen für Newton Stellung. Es treten, wie bereits angesprochen, gleißende Autoscheinwerfer an die Stelle von „Helios stiller Majestät.“ Die Szene ging zudem auf einem (lebensgeschichtlich vorbelasteten, der tyrannische Vater hatte einst den kleinen Franz dort ausgesperrt, und das auch noch nach der konkurrenzfördernden Geburt von dessen Bruder) Balkon vor sich, der sog. „Palatsche“ und bei Anbruch der Nacht, grundiert von der vergeblichen Hoffnung, dort frische Luft und Freiheit erlangen zu können. Karl suchte ja ständig nach einem Weg, seinen Bewachern, Bruneldas pervertierter „Familie“ mit ihrem französischen Oberhäuptling Delamarche entkommen zu können. Man beobachtet die erwähnte Strassenszene, in der ein Richter seinen - an ordinäre Bestechung rührenden - Wahlkampf betreibt. In dieser Gesellschaft, keine Frage, konnte man nicht Jurist werden, und das waren nun einmal Theodor Körner

(C) Der/die Autor(en) 2021

B. Neumann, Umrisse einer Dritten Kultur im interdisziplinären Zusammenspiel zwischen Literatur und Naturwissenschaft, ELECTRISCHER PROMETHEUS. Umrisse einer Dritten Kultur im interdisziplinären Zusammenspiel zwischen Literatur und Naturwissenschaft, https://doi.org/10.1007/978-3-662-63204-8_4 
ebenso wie Franz Kafka gewesen. Nicht einmal das Licht hatte hier seine, doch immerhin griechisch hergebrachte, göttlich überirdische Qualitäten zu bewahren vermocht? Der Tatbestand wird freilich in Kafkas Roman nicht diskursiv verkündet. Sondern symbolisch-poetisch evoziert: Eben durch jenes (widergöttliche) Licht, das die gesamte Passage als eine pervertiert ,juristische“ dominiert. Stets gilt: Das Gesetz, wo es der Gleichstellung von Minderheiten dient, hat bei seiner Konzeption das Licht Gottes über sich. Als Karl seine antidiskriminatorische Verteidigung des Heizers vor dem Kapitän begann, blitzten in New Yorks Hafen die hellen Reflexe des „Stahlmantels“ an den dortigen Kriegsschiffe auf: Der gerechte Jurist als Nachfolger des schimmelreitenden, von der Leinwand herableuchtenden Körner, nunmehr selbst wehrhaft glänzend. ${ }^{1}$ Das Licht dagegen, das die Wahl des Richters beleuchtet, kommt weder von "schimmernder Wehr", noch gar vom Himmel herab. Es wird von gepanzerten „Dreadnoughts“, bzw. von Amerikas tayloristisch betriebener Autoproduktion in die Welt gebracht; in eine Welt übrigens, in der damals Tesla gegen Edison kämpfte - und verlor, trotz elektrotechnisch weit überlegener Konzeption. Und das nur deshalb, weil Edisons politische Beziehungen die besseren waren, mit dem Resultat jener qualvollen, exemplarischen, deshalb ausführlich gefilmten Hinrichtung auf dem neu eingeführten „Elektrischen Stuhl“ (zur Demonstration der Gefahren des konkurrierenden Tesla'schen Wechselstroms). Das Ereignis musste vor aller Leute Augen im Kino und an einem farbigen Kriminellen vollzogen werden. Zeitgenössisch amerikanische Lichtspiel-Sensationen mithin, von denen Kafka samt Familie am Frühstückstisch in Prager Tagblatt und Bohemia lesen konnte (und gelesen hat!), was alles dazu beitrug, Edison jenen prominenten Platz in Kafkas Tagebüchern zu sichern, den er innehat. Die Autoscheinwerfer beleuchten grell die Unmöglichkeit, in den USA das zu werden, was Karl als wunschbiographisch inspirierte Figur seines Autors gern werden möchte, und vor dem Kapitän mit seiner Verteidigung des Heizers schon einmal einübt. Die Szenerie wird wie folgt geschildert: "Einzelne, die geschickt in der Menge verteilt waren, hatten Automobillaternen mit äusserst starkem Licht, das sie die Häuser auf beiden Seiten der Strasse langsam auf- und abwärts führten. In Karls Höhe störte das Licht nicht mehr, aber auf den unteren Balkonen sah man die Leute, die davon bestrichen

\footnotetext{
${ }^{1}$ An dieser Stelle sei auf Franziska Schösslers Aufsatz Verborgene Künstlerkonzepte in Kafkas Romanfragment ,Der Verschollene; in: Hofmannsthal-Jahrbuch, Bd. 6, 1998, verwiesen.
} 
wurden, eiligst die Hände an die Augen führen." ${ }^{2}$ Den letzteren ergeht es wie zuvor Arthur Holitscher, Kafkas Vorgänger und Anreger zur Schilderung des allermodernsten Amerika, der in New York, auf dem berühmtesten Dachgarten der Stadt, Kühle und Naturnähe suchte. ${ }^{3}$ Wo der Österreicher folgende AmerikaErfahrung machte: „Es ist unsagbar. Es ist hoffnungslos. Ich setze mich anders herum - plötzlich kaut am Firmament vor mir ein Riesenmaul das neueste Kaugummi. Die Reklame tobt bei Tag und Nacht. Hinter jenen Sternen sitzt jetzt wahrscheinlich der Manager ... und kalkuliert und diktiert in eine Maschine hinein Ziffern, Tobsucht, Gotteslästerung."4 Die blenderische Veranstaltung als ihrerseits ein tief zweifelhafter Umgang mit dem Licht, sozusagen als Fortsetzung der Newton'schen Barbarei, wie Goethe es noch gesehen hatte; aber nun bei Kafka durch neueste, ihrerseits experimentell gewonnene physikalische Erkenntnisse Einstein'scher und später dann Heisenberg'scher Provenienz differenziert, sie markiert in beiden Fällen das Ende aller Hoffnungen für den europäischen Besucher. Jetzt vermag, jedenfalls was Kafkas Karl angeht, nur noch die utopische Lösung zu helfen. Kafkas Abstiegsschilderung zielt mit den Mitteln des Erzählens auf die Demonstration, dass man in den USA keinen Ersatz für die eigene böhmische Heimat zu finden vermag. Dabei blieb der Prager seiner „Vorlage“ bei Holitscher dennoch eng verbunden - in konsequenter Negation. Er hob von ihr den eigenen, differenten Gestaltungswillen deutlich ab: Mit keinem anderen symbolischen Hilfsmittel als eben der von Goethe, aber damals bereits von Maxwell und Einstein inspirierten Lichtmetapher, die ihrerseits auf die neuesten physikalischen Theorien verwies. Der Tatbestand verband den „electrischen Prometheus“ auch und gerade mit dem mesmeristisch geladenen Kleist, wie nun zu zeigen sein wird (jenen Kleist, den Goethe so intensiv abgelehnt hatte, bis in beschädigende Inszenierungen der Kleist'schen Dramen auf der Weimarer Bühne hinein, nicht einmal die Lustspiele des Preussen fanden des Olympiers Gnade). Dieses falsche, gleißende Licht stellt, weiss man um die intertextuelle Szenerie des Verschollenen, eben seine Abhängigkeit von Arthur Holitschers „Vorlage“, eine genuine Erfindung Franz Kafkas dar (und verkörpert

${ }^{2}$ F. Kafka, Der Verschollene, a. a. O., Bd. II, S. 250 in der Ausgabe von Hans-Gerd Koch (s. Anmerkung 38).

${ }^{3}$ Siehe dazu die Arbeit von Wolfgang Jahn, Kafkas Roman ,Der Verschollene; Stuttgart 1965, wo das enge intertextuelle Verhältnis zwischen Holitschers Reportage und Kafkas Roman so erschöpfend wie überzeugend dargestellt wird.

${ }^{4}$ Arthur Holitscher, Amerika. Heute und Morgen. Reiseerlebnisse, Berlin 1912, S. 62. 
deshalb einen signifikanten poetischen Bestandteil im vergleichbaren Symbolfeld von Process, Vor dem Gesetz und Beim Bau der Chinesischen Mauer). Wer hierin eine wesentliche Übereinstimmung zwischen dem Romantiker Kleist und seinem „Blutsbruder“ Kafka erblicken wollte, verstünde im Tiefsten, was beide verband - außer der ironischen Verbindung zwischen antikem Amazonen- und amerikanischen Jiu-Jitsu-Kampf, was nun darzustellen sein wird.

Open Access Dieses Kapitel wird unter der Creative Commons Namensnennung 4.0 International Lizenz (http://creativecommons.org/licenses/by/4.0/deed.de) veröffentlicht, welche die Nutzung, Vervielfältigung, Bearbeitung, Verbreitung und Wiedergabe in jeglichem Medium und Format erlaubt, sofern Sie den/die ursprünglichen Autor(en) und die Quelle ordnungsgemäß nennen, einen Link zur Creative Commons Lizenz beifügen und angeben, ob Änderungen vorgenommen wurden.

Die in diesem Kapitel enthaltenen Bilder und sonstiges Drittmaterial unterliegen ebenfalls der genannten Creative Commons Lizenz, sofern sich aus der Abbildungslegende nichts anderes ergibt. Sofern das betreffende Material nicht unter der genannten Creative Commons Lizenz steht und die betreffende Handlung nicht nach gesetzlichen Vorschriften erlaubt ist, ist für die oben aufgeführten Weiterverwendungen des Materials die Einwilligung des jeweiligen Rechteinhabers einzuholen.

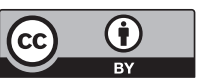

